LBL -16819

Presented at the Conference on Manifestations of

DE84 005199

Hadron Substrupture in Nuclear Physics,

Bloomington, IN, October 19-21, 1983

coNF-8.3 10245--1

\title{
MESON RADIATION FROM QUARK-GLUON PLASMA
}

T. Matsui, B. Banerjee, and N. K. Glendenning

\author{
Nuclear Science Division \\ Lawrence Berkeley Laboratory \\ University of California \\ Berkeley, CA 94720
}

\section{DISCLAIMER}

\begin{abstract}
This report was prepared as an account of work sponsored by an agency of the United States Government. Neither the United States Government nor any agency thereof, nor any of their employees, makes any warranty, express or implied, or aseumes any legal linbility or responsibility for the sccuracy. completeness, or usefulness of any information, apparatus, product, or process discloced, or represents that its use woukd not infringe privately owned rights. Reference hercin to any specific commercial product. process, or service by trade name, trademark, manufacturer, or ofherwise does not necesuarily constitute of jmply its endorsement, recom. mendation, or favoring by the Unitod States Government or any agency thereof. The views and opinjons of authors expresued herein do not necessarily state or reflect thoee of the United States Government or any agency thereof.
\end{abstract}

This work was supported by the Director, office of Energy Research Division, of Nuclear Physics of the Dffice of High Energy and Nuclear Physics of the U.S. Department of Energy under Contract No. DE-ACO3-76SF00098. 


\title{
MESON RADIATION FROM QUARK-GLUON PLASMA
}

\author{
T. Matsui, B. Banerjee, * and N. K. Glendenning \\ Nuclear Science Division, Lawrence Berkeley Laboratory \\ University of California, Berkeley, CA 94720
}

\section{ABSTRACT}

Meson formation and radiation at the surface of quark-gluon plasma are studied by making use of the chromolectric flux tube model. The effect of the surface meson radiation on the hydroexpansion of the plasma fluid is also discussed.

\section{INTRODUCTION}

In the recent progress of hadron physics based on QCD, it has appeared very likely that hadrons dissolve into an almost ideal gas of quarks and gluons (quark-gluon plasma i at sufficiently high energy densities, 1-4 the condition which once prevailed in the early universe. Ultrarelativistic heavy ion collis ions may offer an unique opportunity to reproduce such primeval matter in humancontrolied terrestrial laboratories. Several estimates of the expected energy deposition in the very energetic central nuclear collision $(\sqrt{5} \geq 20 \mathrm{GeV}$ per nucleon) confirmed that the energy density will certainly exceed the critical value $\left(-2 \mathrm{GeV} / \mathrm{fm}^{3}\right)$ which is required to complete the phase transition, both in the fragmentation (nuclear fire ball) region 5 and in the central rapidity region. 6,7 once a quark-gluon plasma is formed in such a process the high internal pressure and temperature will cause its rapid disassembly. Thus, to make a reliable prediction for possible signals of plasma formation a dynamical description of the plasma evolution is of crucial importance.

One facet of the time evolution of quark-gluon plasma has been studied by several authors 6-9 who use relativistic hydrodynanics to describe the plasma expansion. As the plasma expands it will cool, and then the conditions for condensation back to the hadronic phase will be attained. In the present work we focus on another facet of the plasma evolution, the formation and radiation of mesons at the surface of a hot plasma. The surface meson radiation would play two important roles. First, it may carry some information about the pre-freezeout stage of the plasma evolution. 10,2 Second, it causes a pressure decrease at the surface that works against the expansion. In the extreme, plasma may evaporate very rapidly by surface meson radiation without collective expansion. Such an extreme scenario was proposed recently by Danos and Rafelski with a two-parameter model of surface radiation. 11 However, in their model there is no dynamical description of meson formation at the plasma surface. Therefore it was very unclear how in itial quark

*Permanent address: Tata Institute for Fundamental Research, Bombay, India. 
degrees of freedom are converted into mesonic degrees of freedom and how color confinement works in such a process. Quite independently we have studied the mechanism of surface meson radiation by fully employing the chromolectric flux tube model. 12 We found that the parametrization of Danos and Rafelski is quite unsatisfactory and is actually incompatible with a proper treaiment of color confinement. In the following we shall briefly recajitulate our treatments and findings.

\section{A SCENARIO OF SURFACE MESON RADIATION}

Our scenario of the surface meson radiation consists of three stages. The plasma may be viewed as a big MIT bag occupied by the gas of almost free quarks and gluons. The surface of the plasma is identified with the bag surface, which separates the perturbative QCD vacuum in the plasma from the true nonperturbative vacuum outside of the plasma. Then the meson radiation is initiated by the emission of a single quark from the plasma surface (Fig. 1a). This is caused by the thermal motion of quarks, and the flux of the quark is therefore characterized by the surface temperature. This

leading quark cannot escape

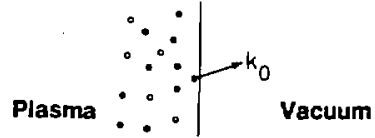

(a)

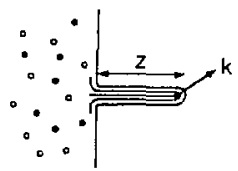

(b)

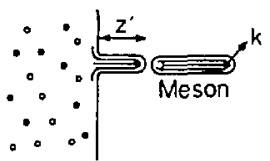

(c)

$\times B \cdot 8310-617$

Figure 1. Three stages of the meson formation process at the. plasma surface. from the plasma alone because of color confinement. The next stage of our scenario is the formation of the chromoelectric flux tube that connects the leading quark to the plasma surface (Fig. Ib). Now the leading quark suffers a very strong attractive interaction with the plasma, which is characterized by the string tension of the flux tube. It is decelerated and will eventually be reabsorbed by the plasma unless the flux tube breaks. The fission of the flux tube can occur through the quantum tunneling of a virtual q⿳亠口冋 pair, formed in the uniform color electric field inside the tube, into a real state (Fig. 1c). The piece of flux tube disconnected from the plasma forms a meson and is emitted from the plasma Its energy and momentum are determined by the initial energy and momentum of the leading quark and the space-time 
position where q $\bar{q}$ pair creation occurs. This mechanism of hadronization has been commonly used to describe particle production in high energy $\mathrm{e}^{+} e^{-}$annihilation. Although in our problem the average momentum of quarks is not so high $\left(k_{0} \sim T-200 \mathrm{MeV}\right)$, this picture qualitatively describes the fate of the high momentum component of quarks (antiquarks) in the thermal distribution. The contiribution of the low momentum quarks to this hadronization process is suppressed, as we shall see. This supports our idealization of a smooth plasma surface. One may consider another mechan ism for the hadronization at the plasma surface, i.e., the coalescence of a quark-antiquark pair that already exists in the plasma. We do not deal with this process here, because at the expected plasma temperature the thermal flux of quarks and antiquarks with specified color charge is very small.

\section{FLUX TUBE FORMATION AT THE PLASMA SURFACE}

As a quark comes out of the plasma, its kinetic energy is gradually converted into the field energy of the chromoelectric: field, thus decelerating the quark. The energy stored in the flux tube per unit length is given by the string tension $\sigma$, which is empirically known to be o $-0.9 \mathrm{GeV} / \mathrm{fm}$ from Regge slope. We now assume that the flux tube connects the leading quark to the plasma by the snortest path. Then the classical trajectory of the quark is readily obtained by integrating $\mathrm{dr} / \mathrm{dt}=\mathrm{k} / \mathrm{E}$, where the energy $E$ and the transverse (parallel to the surface) momentum $k_{T}$ of the leading quark at time $t$ after its emission from the surface are related to their initial values by the conservation laws:

$$
E+E_{S}=E_{0}, k_{T}+k_{S}=k_{T O} \text {. }
$$

Here $E_{S}=\sigma z\left(1-v_{T}\right)^{-1 / 2}$ and $k_{S}=\sigma z v_{T}\left(1-v_{T}\right)^{-1 / 2}$ are the energy and the momentum of the flux tube with length $z$ which is moving parallel to the surface with velocity $v_{T}$. The resultant longitudinal motion of the quark is described by:

$$
\begin{gathered}
z=\left(E_{z 0}-E_{z}\right) / \sigma \\
k_{z}=k_{z 0}-\left(E_{z 0} / E_{0}\right) \sigma t .
\end{gathered}
$$

where

$$
E_{0}=\sqrt{k_{0}+m^{2}}, E_{z 0}=\sqrt{k_{z 0}+m^{2}} \text { and } E_{z}=\sqrt{k_{z}+m^{2}} \text {. }
$$

The motion of the quark normal to the surface is decelerated until it stops at time $t_{c}=\left(E_{0} / E_{20}\right)\left(k_{z o} / \sigma\right)$. Thereafter it is accelerated back to the plasma. During this period the longitudinal quark momentum decreases linearly as a function of time $t$, whereas the transverse velocity of the quark and the flux tube remains constant $\left(v_{T}=v_{T O}=k_{T o} / E_{0}\right)$. The field energy stored in the 
flux tube can be further converted into a new $q \bar{q}$ pair through the quantum tunneling, causing the fission of the flux tube. If such a pair is created at a distance $z^{\prime}$ from the surface and at a time $t$, a meson consisting of the leading quark together with the antiquark of the new created pair is formed with the energy $E^{M}$ and the longitudinal momentum $k$ which are given by:

$$
\begin{aligned}
& E^{M}=E_{0}-\left(E_{0} / E_{Z O}\right) a z^{\prime}, \\
& k_{Z}^{M}=k_{Z O}-\left(E_{Z O} / E_{0}\right) \sigma t
\end{aligned}
$$

where $\left(E_{0} / E_{z_{0}}\right){ }^{\prime} z^{\prime}$ is the energy of the leftover flux tube which is Lorentz-boosted by the transverse motion.

\section{IV. $q \bar{q}$ PAIR CREATION AND FISSION OF THE FLUX TUBE}

The $q \bar{q}$ pair creation in the constant color electric field is quite analogous to the problem considered by Schwinger 13 relating to the vacuum persistency against $\mathrm{e}^{+} \mathrm{e}^{-}$pair creation in a constant electric field. This old and rather academic problem has been recently found by Casher, Neuberger and Nussinov 14 to be of practical importance in the context of QCD. They applied Schwinger's result directly to multiple hadron production in high energy $\mathrm{e}^{+} \mathrm{e}^{-}$annihilation. In Schwinger's calculation, the effect of the interaction was included only between each of the created pair and the external field, and therefore it was implicitly assumed that the electromagnetic interaction between the members of the pair is negligible compared to that with the external field. Although this approximation may be somewhat justified in Schwinger's problem, this is certainly not the case in our flux tube problem where the field between the members of the created pair is exactly identical in strength to the original field and is oppositely directed, thereby canceling the original field. We derived a new result for the qq pair creation rate in the flux tube using an improved energy balance relation in the intermediate virtual state, which includes the effect of the mutual interaction of new q $\bar{q}$ pair. 16 our result for the probability of making a $q \vec{q}$ pair per unit four volume is given by

$$
p=\frac{\sigma^{2}}{4 \pi^{3}} \sum_{\text {Flavor }} \sum_{n=1} \frac{1}{n^{2}} \exp \left(-\frac{\pi m_{f}^{2} n}{\sigma}\right)
$$

If we use current quark masses as in Ref. 15, then this probability is well approximated by $p=\sigma^{2} /(12 \pi)$. 
The fission probability of the flux tube at time $t$ and at position $z$ ' with infinitesimal space-time interval is now given by $d^{2} p=p A R(t) d z^{\prime} d t$ where $A$ is the cross section of the flux tube and $R(t)=1-P(t)$ is the attenuation factor, which selects out the unwounded flux tube. Noting the previous relation between the space-time position of the fission and the energy-momentum of the meson, Eq. (3), we can rewrite this fission probability as a function of meson coordinates, $\mathrm{k}^{\mathrm{M}}$ and $\mathrm{E}^{\mathrm{M}}$. In the massless quark 1 imit, we have a very simple result

$$
d^{2} p=\exp \left(-\frac{k_{0}\left(k_{z}-k_{z 0}^{M}\right)^{2}}{2 k_{z 0} k_{c}^{2}}\right) d\left(\frac{k_{z}^{M}}{k_{c}}\right) d\left(\frac{E^{M}}{k_{c}}\right)
$$

where all energy momentum variables are scaled by single parameter

$$
k_{C}=\sqrt{\sigma^{2} / \mathrm{PA}}=\sqrt{12 \pi / A}=\sqrt{12} / R
$$

and $R$ is the radius of the flux tube. For $R=0.5(1.0) \mathrm{fm}$, we have ${ }^{k} c_{c}=1.38(0.69) \mathrm{GeV}$, which is large compared to the re$\cdots$ ant temperature that we consider. The physical meaning of this parameter is obtained if one computes $\mathrm{dP} / \mathrm{dk} Q$ from eq. (5). The result is plotted in Fig. 2, which shows that the most probable momentum $k_{m}$ of the meson is smaller than the initial quark momentum $k_{o}$ by the amount $k_{c}$. Thus $k_{c}$ characterizes the effect of confining force which suppresses large momentum transfer to mesons and which prohibits the low momentum quarks with $k_{0} \leqslant k_{c}$ to participate in this process.

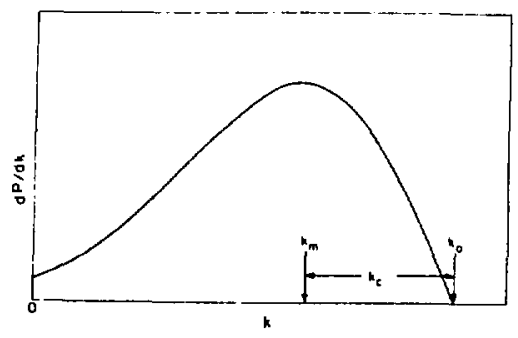

Fig. 2 Momentum distribution of the meson for initial quark momentum $k_{z o}>k_{c}$. 


\section{RADIATION SPECTRUM AND BACKWARD RADIATION PRESSURE}

We are now in the position to compute the spectrum of mesans emitied from the plasma surface. The meson flux is given by the thermal flux of the quarks and antiquarks multipliad by the fission probability of the flux tube. Because the fission probability has been computed as a function of meson momentum and energy, we can derive the meson spectrum by integrating over the initial quark momentum

$$
\begin{aligned}
& \frac{d^{2} \text { (Meson Flux) }}{d k_{z}^{M} d E^{M}}=\frac{r}{(2 \pi)^{3}} \int d^{3} k_{0} e^{-E_{0} / T k_{20}} \frac{E_{0}}{E^{M}}\left(k_{z}^{M}, k_{0}\right) \frac{1}{k_{c}^{2}} \\
& \quad x \in\left(\sqrt{k_{z}^{M^{2}}+m^{2}}<\left(E_{z 0} / E_{0}\right) E^{M}\right) \theta\left(\left(E_{z 0} / E_{0}\right) E^{M}<E_{z 0}\right) \\
& \quad x \theta\left(k_{z}^{M}<k_{z 0}\right) \theta\left(k_{z}^{M}>0\right)
\end{aligned}
$$

where step functions make several constraints for the kinematically al lowed processes. Here we simply use the Boltzmann distribution function with zero chemical potential and $r=r_{c} r_{s} r_{f} \cdot 2=24$ is the degeneracy factor including quark-antiquark degrees of freedom.

We first apply this result to estimate the influence of meson radiaton on the hydroexpansion. Because the radiating mesons carry away a certain amount of momentum that otherwise contributes to the quark pressure acting on the plasma surface, there is a pressure decrease at the surface. The backward radiation pressure can be estimated by

$$
P_{r a d}=\int_{0} d k_{z}^{M} \int_{E_{\pi}} d E^{M} \frac{d^{2}(\text { Meson Flux) }}{d k_{z}^{M} d E^{M}}\left(2 k_{z}^{M}\right)
$$

where we introduced the pion mass cutoff $E_{\pi}=\sqrt{k \xi+m_{\pi}^{2}}$ for the minimum energy of mesons. In the case, $m_{\pi} \ll T \ll k_{C}$, we have a simple analytic formula, $\mathrm{Prad}_{\text {ra }}=5 / 2\left(T / \mathrm{k}_{\mathrm{C}}\right)^{2} \mathrm{PQ}$, where $\mathrm{PQ}_{\mathrm{Q}}=$ $\left(r / \pi^{2}\right) \cdot T^{4}$ is the quark-antiquark pressure in the massiess

Boltzmann gas approximation. Figure 3 shows the numerical result of the ratio of the radiation pressure to the quark pressure as a function of surface temperature. One sees that this ratio is less than 20 percent at relevant temperatures, indicating that the meson radiation due to this pracess is a minor perturbation on the hydroexpansion of the plasma. 


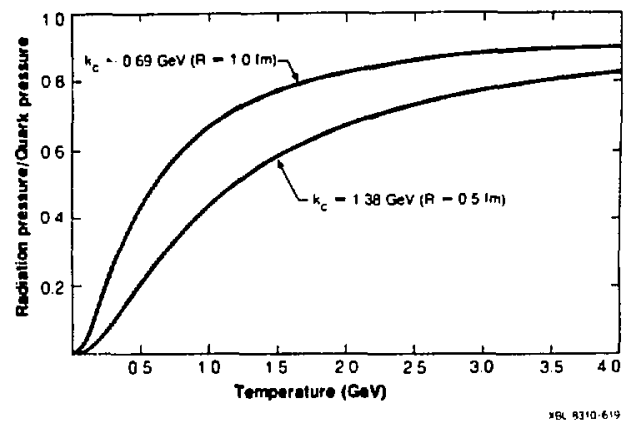

Fig. 3. The ratio of backward radiation pressure to the quark pressure as a function of plasma temperature.

\section{SURFACE TEMPERATURE AND SURFACE MOTION}

To determine surface temperature and surface motion we invoke the macroscopic energy-momentum conservation $1 \mathrm{aw}, a_{\mu} T^{\mu \nu}=0$, where the energy-momentum tensor $T^{\mu \nu}$ may be written as $T_{I N}^{\mu \nu}=P g^{\mu \nu}+$ $(P+E) U^{\prime \prime} y^{\nu}$ in the collis ion dominated plasma region, while outside of the plasma it takes a form, TouT = Bguv + Trad. Here $\varepsilon$, $U^{\mu \nu}$ are the energy density and the velocity of the plasma fiuid, and $B$ and Trad. denote the bag constant and the radiation energy-momentum tensor respectively. Then the energy-momentum conservation law gives a set of equations which determine the hydrodynamical motion of the plasma with a surface boundary condition:

$$
\begin{aligned}
& a_{\mu} T_{I N}^{\mu \nu}=0: \text { hydrodynamics } \\
& T_{I N}^{\mu \nu} n_{\mu}=T_{\text {OUT }}^{\mu \nu} n_{\nu}: \text { surface boundary condition }
\end{aligned}
$$

where $n_{\mu}$ is a unit space-like four vector normal to the surface world sheet. In the rest frame of the surface Eq. (10) gives a pressure balance equation at the surface;

$$
P_{\text {plasma }}\left(T_{S}\right)=B+P_{\text {rad. }}\left(T_{S}\right)
$$

Because the radiation pressure $P$ rad. has been determined as a function of surface temperature $T_{S}$ by Eq. (8) this equation determines the surface temperature of the plasma as a function of external bag pressure. For a small flux tube radius $(R=0.5 \mathrm{fm})$, it turns out to be a good approximation to neglect the radiation pressure, yielding 


$$
T_{s}=0.7 \mathrm{~B}^{1 / 4}
$$

for $U, d$ and gluon plasma. This formula gives $T_{S}=140-200 \mathrm{MeV}$ for $\mathrm{B} 1 / 4=200-280 \mathrm{MeV}$. The above analys is also indicates that the plamsa surface may be slightly supercooled ( $T_{S}<T_{C}$ ) because the critical temperature $T_{C}$ is determined by $P_{p l}\left(T_{C}\right)=B+P_{h a d}\left(T_{C}\right)$ with thermal hadron pressure $P_{\text {had. }}$. Which exceeds $P_{\text {rad. at low tem- }}$ perature.

The surface $\pi$, tion can be determined by solving the hydrodynamic equation (9) with surface boundary condition (10) (or (11)). Since it requires an extensive numerical study to solve this problem in the general geometrical condition, we consider here only one-dimensional flow where a simple analytic solution exists. In this case our problem is reduced to a problem of gas flow in a semi-infinite pipe terminated by a piston which begins to move with constant velocity. The resultant flow is a similarity flow which consists of two steady flow regions and a rarefaction wave in between. 17 The pressure profile of the fluid is illustrated in Fig. 4. The fluid is at rest in the interior region (I) with high constant pressure $P_{0}$ and is accelerated by the pressure gradient of the rarefaction wave (II) to get the velocity $v_{S}$ of exterior steady flow (III) where the pressure becomes constant again, $P_{S}$ B. The acceleration due to the rarefaction wave can be estimated by a formu 1 a 18

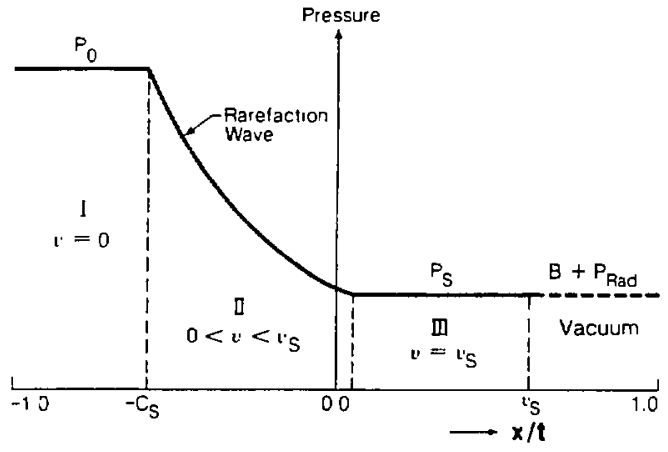

19. Aาt 6

Fig. 4. Pressure profile of one-dimensional relativistic similarity flow which cons ists of two steady flow region (I and III) and a rarefaction wave (II). 


$$
y_{S}=\tanh ^{-1} v_{s}=\int_{P_{S}}^{P_{0}} \frac{d P}{c_{s}(P+\varepsilon)}=\sqrt{3} \ln \left(T_{0} / T_{s}\right)
$$

where $c_{s}=\sqrt{\mathrm{dP} / \mathrm{d}_{\varepsilon}}$ is the sound velocity and, in deriving the last equality, we used the Stephan-Boltzmann equation of state. This formula predicts the sumface rapidity $y_{s}=0.7$ for $T_{0} / T_{s}$ $=3 / 2$. The radiation spectrum obtained in the previous section should be corrected by the Doppler shift due to this surface motion.

\section{PION RAPIDITY DISTRIBUTION}

Finally, we compute the rapidity distribution of pions which are produced by the surface radiation. Because the mesons which are formed by the flux tube fission have a continuous spectrum of invariant mass $M_{S}$ that is greater than the pion mass, they will eventually decay into pions, photons, and so on. If we take the flux tube nodel seriously, we have to compute the cascade decay of long flux tubes until we get only pions or other stable narticles. Instead of doing this, we compute the dominant decay mode $i y$ assuming the statistical distribution in the phase space. This is done analytically up to three pion decay. For large $M_{5}$, we use empirical inclusive cross section of $\mathrm{e}^{+} \mathrm{e}^{-} \rightarrow q \bar{q} \rightarrow \pi+x_{\text {, which }}$ exhibits very simple scaling nature at high energies. 19 3esides the initial electromagnetic process, this process is identical to the decay of a high energy string. Once the rapidity distribution of pions, $d N_{\pi} / d y$, is obtained on the rest frame of the string ( = heavy meson), the rapidity distribution of pion flux on the rest frame of the plasma surface can be computed by folding this by the rapidity-invariant mass distribution of the meson (= broken string flux

$$
\frac{d(\text { Pion Flux) }}{d y}=\int d y_{S} d M_{S} \frac{d^{2} \text { (Meson Flux) }}{d y_{S} J M_{S}} d y^{\prime} \frac{d N_{\pi}}{d y^{\top}} \delta\left(y-y_{s}-y^{\prime}\right)
$$

where $y_{S}=\tanh ^{-1}\left(k_{2} / E^{M}\right)$ is the rapidity of the broken siring normal to the plasma surface. The numerical result of the rapidity distribution of pion flux at $T_{S}=200 \mathrm{MeV}$ is shown in Fig. 5 where the rapidity distributions of thermal (black body) quark-antiquark flux and the broken string flux are also plotted. We see that al though the quark confinement reduces the number of strings and pions from that of quarks and antiquarks, almost the same number of pions are still produced as the black body radiation of pions at 
the same temperature. This is not so surprising because the large number of degrees of freedom (or large entropy) are liberated in the plasma phase; $\gamma=8 \gamma_{\pi}$ where $\gamma_{\pi}=3$ is the pion degeneracy factor. Note that the peak of the rapidity distribution is sinifted downward showing a characteristic feature of the pion spectrum produced in this particular process. Pion rapidity in the laboratory frame is obtained from this resuit by simply shifting $y \rightarrow y_{L a b .}=$ $y+y_{S}$ where the surface rapidity $y_{S}$ is determined by Eq. (13).

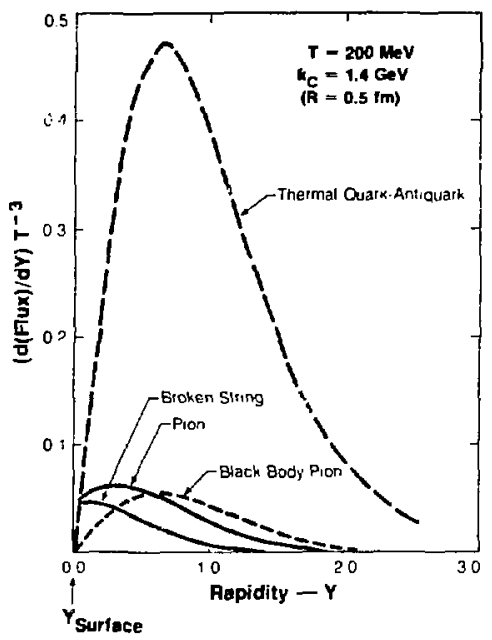

Fig. 5. Rapidity distribution of pions which are produced by the decay of heavy mesons is compared with that of initial quark-antiquark, broken string, and black body pion radiation.

VIII. CONCLUDING REMARKS

We have studied the meson emission from a quark-givoir plasma surface in the framewro of the chromelectric flux tube model. It was found that in the present scenario only high momentum quarks in the plasm make a significant contribution to the surface hadronization because the encigy scale which characterizes the fission of 
the flux tube due to q $\bar{q}$ pair creation is fairly large compared to the plasina temperature. Hence the resultant mesun radiaton turns out to be a minor perturbation on the hydroexpansion of the plasma fluid. It should be noted that this result is consistant with our idealization of a smooth plasma surfare.

The plasma surface, as a whole, is stabilized by the pressure b.al ance beiween internal plasma pressure and the external bag pressure plus the radiation pressure. This requirement determines the surface temperature. We observed that this is one of the consequences of the energy-momentum conservation law, which can be used to determine the hydrodynamical evolution of the interior f?asma fluid. The above picture is, however, somewhat different from what is commonly conceived of in the macroscopic descriptions of the plasma evolution. Usually it is implicitly assumed that the chemical reaction from deconfined quarks and gluons in the plasma to hadrons takes place very rapidiy and smoothly at the plasma surface, and that the plasina core is therefore surrounded by a dense hadror cas which is also described by the hydrodynamics until its temperature reaches the freezeout temperature, $T_{f} \sim m_{\pi}$. In the present descripition, mesons stream out of the plasma surface freely without interacting with each other oecause of the very slow chemiral reaction rate at the plasma surface.

The surface radiation we described in this paper is a highly nonequilibrium process. Nevertheless the pion spertrum obtained in the present calculation shows a strong similarity in its shape as well as its intens ity to the slack body radiation spectrum of the thermal pion gas whose temperature is equivalent to the surface temperature of the plasma. As a notable distinction from the black-body radiation we observed a backward shift of the peak in the rapidity distribution. However the who?e spectrum is also shifted forward by the Doppler effect due to the surface motion which is caused by the hydrodynamical motion of the interior plasma fluid. We constructed an analytic solution to the one-dimensional hydrodynamics with a surface boundary condition and found a significant acceleration of the plasma surface. To make a more accurate estimate for the radiation spectrum it is certainly required to have a more realistic description of the initial corditions of the hydrodynamics and the resultant motion of the plasma surface.

\section{ACKNOWLEDGMENTS}

The authors are pleased to thank G. Baym, M. Gyulassy, A. Hosoya and L. Van Hove for beneficial discussions and usefu? suggestions.

This work was supported by the Director, Office of Energy Research Division, of Nuclear Physics of the Office of High Energy and Nuclear Physics of the U.S. Department of Energy under Contract No. DE-AC03-76SF00038. 


\section{REFERENCES}

1. J. C. Collins and M. J. Perry, Phys. Rev. Lett. 34, 1353 (1975).

2. E. V. Shuryak, Phys. Rep. 61, 72 (1980).

3. D. J. Gross, R. D. PisarskT, and L. F. Yaffe, Rev. Mod. Phys. 53, 43 (1981).

4. "Quark Matter Formation and Heavy Ion Collisions", Proceedings of the Bielefeld Workshop, Ed. M. Jacob and H. Satz, World Scientific (1982).

5. R. Anishetty, P. Koehler, and L. McLerran, Phys. Rev. D22, 2793 (1980).

6. J. D. Bjorken, Phys. Rev. D27, 140 (1983).

7. M. Gyulassy and T. Matsui, $\overline{L B L}$ report LBL-15947, Phys. Rev. D in press.

8. K. Kajantie, R. Raitio, and P. V. Ruuskanen, Nucl. Phys. B222, 152 (1983).

9. G. Baym, J.-P. Blajzot, W. Czyz, B. L. Friman, and M. Soyeur, Nuc1. Phys. A (1983) in press.

10. N. K. Glendeñning and K. Karant, Phys. Rev. C21, 1501 (1980).

11. M. Danos and J. Rafelski, Phys. Rev. D27, 671 (1983).

12. B. Banerjee, N. K. Glendenning, and T. Matsui, Phys. Lett. 127B, 453 (1983).

13. J. Schwinger, Phys. Rev. 82, 664 (1951).

14. A. Casher, H. Neuberger, and S. Nussinov, Phys. Rev. 020, 179 (1979).

15. E. G. Gurvich, Phys. Lett. 87B, 386 (1979).

16. N. K. Glendenning and T. Matsui, Phys. Rev. D28, (1983) in press.

17. L. D. Landau and E. M. Lifshitz, Fluid Mechanics (Pergzrion, 1959): see also Ref. 9 for relativistic extension of the Riemann solution of the rarefaction wave.

18. See L. D. Landau and E. M. Lifshitz, Toc.cit., p. 503.

19. R. Brandelik, et al., Phys. Lett. 67B 358 (1977); 89B, 418 (1980). 
This report was done with support from the Department of Energy. Any conclusions or opinions expressed in this report represent solely those of the author(s) and not necessarily those of The Regents of the University of California, the Lawrence Berkeley Laboratory or the Department of Energy.

Reference to a company or product name does not imply approval or tecommendation of the product by the University of California or the U.S. Department of Energy to ine exclusion of others that may be suitable. 